OPEN ACCESS

Edited by:

Li Wang,

Institute of Psychology, Chinese Academy of Sciences (CAS), China

Reviewed by:

Daniela Adamo,

University of Naples Federico II, Italy Arturo Garay,

Centro de Educación Médica e Investigaciones Clínicas Norberto Quirno (CEMIC), Argentina

*Correspondence: Ruihua Wei rwei@tmu.edu.cn

Specialty section: This article was submitted to Public Mental Health, a section of the journa

Frontiers in Psychiatry

Received: 26 October 2021 Accepted: 13 December 2021

Published: 05 January 2022

Citation:

He Q, Chen Z, Xie C, Liu L and Wei R (2022) The Association Between Dry Eye Disease With Depression, Anxiety and Sleep Disturbance During COVID-19.

Front. Psychiatry 12:802302. doi: 10.3389/fpsyt.2021.802302

\section{The Association Between Dry Eye Disease With Depression, Anxiety and Sleep Disturbance During COVID-19}

\author{
Qing He, Zhuo Chen, Caiyuan Xie, Lin Liu and Ruihua Wei*
}

Tianjin Key Laboratory of Retinal Functions and Diseases, Tianjin Branch of National Clinical Research Center for Ocular Disease, Eye Institute and School of Optometry, Tianjin Medical University Eye Hospital, Tianjin, China

Objective: This study aimed to investigate the relationship between dry eye disease (DED) with anxiety and depression. Additionally, the mediating effect of sleep quality on this relationship was explored.

Methods: 321 patients with DED were recruited from Tianjin Medical University Eye Hospital clinic and surveyed using demographic questionnaires, the Ocular Surface Disease Index (OSDI), Hospital Anxiety and Depression Scale (HADS), and Pittsburgh Sleep Quality Index (PSQI). Regression analysis and the bootstrap method were used to investigate the influence of sleep on the relationship between DED, anxiety and depression.

Results: Among the patients with DED, 86 (26.79\%), 85 (26.48\%), and 54 (16.82\%) patients presented with anxiety, depression, and both anxiety and depression respectively. The OSDI and PSQI score were positively correlated with depression and anxiety (all $p<0.01$ ). The direct effects of OSDI on depression and anxiety were significant $(P<0.01)$. Additionally, the bootstrap test showed significant mediating effects of subjective sleep quality [95\% Cl [0.003-0.016] (depression); [0.001-0.011] (anxiety)] and sleep latency [95\% Cl [0.001-0.010] (depression); [0.001-0.008] (anxiety)]. These results indicated that the severity of DED symptoms, as measured by the OSDI score, affected anxiety and depression through a direct and an indirect pathway mediated by subjective sleep quality and sleep latency.

Conclusions: The results indicated that there was a significant correlation between DED and anxiety and depression. Moreover, subjective sleep quality and sleep latency were a mediator of the relationship between DED symptoms and anxiety and depression.

Keywords: dry eye disease, anxiety, depression, dry, sleep, COVID-19

\section{INTRODUCTION}

Since December 2019, coronavirus disease 2019 (COVID-19) has swept the world and has been declared a global public health emergency by the World Health Organization. COVID-19 is an infectious disease caused by severe acute respiratory syndrome corona virus 2 (SARS-COV-2) with droplets and contact as the main modes of transmission (1).COVID-19 is characterized by high 
infectivity and mortality. The cumulative number of confirmed cases worldwide was $200,174,883$ and of deaths were $4,255,892$, respectively, until August 5, 2021.

The widespread and rapid dissemination of COVID-19 has caused serious mental health stress to the public, such as anxiety, depression, and sleep problems (2-4). Meanwhile, during COVID-19 outbreak, individuals' isolation at home and the use of electronic devices have increased greatly (5).Long-term use of electronic products will inevitably cause eye diseases, such as dry eyes disease, myopia $(6,7)$.

Previous research (8) found ocular problems in COVID-19 patients, with conjunctival congestion as the primary symptom (9), while eye pain, photophobia and dry eye were also reported (10). Dry eye disease (DED) is a common ocular surface disease, with patients complaining of discomfort including dryness, foreign body sensation, burning sensation, photophobia, and eye pain, which can affect their the quality of life $(11,12)$. DED patients with chronic eye discomfort may also experience emotional problems such as anxiety and depression (13-15).

Several studies (16-19)have found the prevalence of depression in dry eye patients to be around $25-53.7 \%$ and anxiety around $39-63.6 \%$. The severity of signs and symptoms in patients with DED did not change concomitantly. Kim et al. (20) found that the discomfort of DED and anxiety-depression were correlated; however, several studies concluded the opposite (2123). Since the relationship between the symptoms of DED and anxiety and depression is unclear, it is crucial to conduct further research. Moreover, sleep disorders are another serious problem in patients with DED. In addition, patients with DED are more likely to have sleep problems than other eye diseases (18). Wu et al. (24) have found that DED patients with sleep disorders are more likely to experience anxiety and depression. Ayaki (25) found that subjective sleep quality was closely associated with anxiety and depression in women with DED. Sleep and mental problems in patients with DED have been the main interest of many researchers, but the precise association between the different components of sleep and anxiety and depression is not clear.

Various studies have found an increase in anxiety, depression, and sleep disorders during the COVID-19 epidemic (4, 26, 27). However, the relationship between sleep and mood problems in individuals with DED during the COVID-19 pandemic is unclear. Therefore, we want to investigate whether there is a correlation between DED, sleep, anxiety and depression. Furthermore, we try to explore the mediating effect of sleep on the relationship between DED and anxiety and depression in patients during the COVID-19 pandemic.

\section{MATERIALS AND METHODS}

The 321 patients with DED were recruited between May and July 2021 at Tianjin Medical University Eye Hospital in China. Approval was obtained from the Medical ETHICS Committee of Tianjin Medical University Eye Hospital. The procedures used in this study adhered to the tenets of the declaration of helsinki. informed consent was obtained from all participants included in the study.

Participants were literate adults aged $>18$ years with an Ocular Surface Disease Index (OSDI) score of $\geq 13$ points. We excluded patients who had complications from anterior ocular segment diseases except for DED, patients with a history of ophthalmic surgery in the last 3 months, patients with severe glaucoma, bilateral cataract, and exclude systemic diseases such as autoimmune diseases, severe cardiopulmonary diseases, allergic diseases, and neurologic or psychiatric disorders. Previously diagnosed anxiety and depression were also excluded. In addition, patients who received anti-allergy drugs and contraceptives were excluded. Patients with an alcohol and drug dependence history, those taking anti-anxiety and antidepressant drugs, with serious medical conditions that prevented them from completing the questionnaire, who were unable to care for themselves, severely illiterate, and pregnant and lactating women were also excluded. Demographic and medical data were collected.

\section{Evaluation of DED}

All participants completed the OSDI questionnaire, a selfadministered questionnaire that assesses the severity of selfreported DED. Based on the total OSDI score, each participant's condition was classified as normal (0-12 points), mild (13-22 points), moderate (23-32 points), and severe (33-100 points). A score of $\geq 13$ points led to a diagnosis of DED.

\section{Sleep Quality Assessment}

Patients' sleep quality in the last 1 month was measured using the Pittsburgh Sleep Quality Index (PSQI). It consists of seven dimensions, namely subjective sleep quality, sleep latency, sleep duration, habitual sleep efficiency, sleep disturbance, use of sleep medicine, and daytime dysfunction. Each dimension is scored on a scale of $0-3$, and dimension scores are cumulated to a total PSQI score ranging from 0 to 21 , with higher total scores indicating poorer sleep quality. Patients with total scores $>7$ were considered to have poor sleep.

\section{Emotion Status Assessment}

The Hospital Anxiety and Depression Scale (HADS) questionnaire was created by Zigmond and Snaith (28) to screen for anxiety and depression among patients in general hospitals. The scale contains 14 items, divided into two subscales containing seven items each to assess depression and anxiety. The scale is scored on a 4 -point scale (0-3), and the cut-off points for anxiety and depression are total scores of 8 points. HADS has good reliability and validity values (29), and is widely used to screen for anxiety and depression.

\section{Statistical Analysis}

Statistical analyses were performed using the SPSS software (version 23.0; IBM Corp., Armonk, NY, USA).The normality of each continuous variable was tested. Continuous variables that conform to the normal distribution are expressed as Mean \pm $\mathrm{SD}$, and those that do not are expressed as median (IQR).Using independent $t$-test or $\chi 2$ test for continuous and categorical 
variables, respectively. When the theoretical frequency $(\mathrm{T})$ was $1 \leq \mathrm{T}<5$, a corrected $\chi 2$ test was used.We used Spearson's correlation to analyze the associations between the scores on the OSDI, PSQI and subscales, and HADS. Multiple linear hierarchical regression models were applied to test the mediating role of total PSQI and subscales scores in the relationship of DED with anxiety and depression. Prior to the mediation analysis, all continuous variables were pooled to eliminate multicollinearity. We used $B$ as an unstandardized regression coefficient to describe the significant of direct and indirect effects, Beta as a standardized regression coefficient to describe the weight of them. The mediating role of sleep quality was further tested using a bootstrap analysis of 5,000 samples. All tests were two-sided, and statistical significance was set at $p<0.05$.

\section{RESULTS}

This study included 321 patients with DED (89 men and 232 women).The age of them was $48.41 \pm 15.15$. General patient characteristics are presented in Table 1. We found no difference in sleep between men and women with DED. Menopausal women were more likely to have poor sleep $(p<0.05)$.In addition, age and education levels were also affected factors of sleep $(p<0.05)$.

Among participants, 86 (26.79\%), 85 (26.48\%), and 54 (16.82\%) presented with anxiety, depression, and both anxiety and depression, respectively. A total of $52(16.20 \%)$ patients reported good sleep, and 269 (83.80\%) reported poor sleep. In addition, DED patients who had poor sleep were more likely to be anxious and depressed (Table 2).

The mean OSDI score was $45.90 \pm 15.90$ points. We found that $243(75.70 \%)$ patients with DED were categorized as severe according to the OSDI score, while 18 (5.61\%) and 60 (18.69\%) patients were categorized as mild and moderate, respectively.

The results of the correlation analyses are presented in Table 3. The OSDI score was significantly correlated with anxiety and depression, as well as with PSQI total score and subjective sleep quality and sleep latency. PSQI total score, subjective sleep quality, and sleep latency were significantly correlated with anxiety and depression.

Hierarchical regression analyses (Tables 4, 5) revealed that more severe DED (i.e., higher OSDI scores) was associated with poorer subjective sleep quality $(B=0.012, p=0.000)$, longer sleep latency $(B=0.009, p=0.027)$, and depressive $(B=0.034$, $p=0.003)$ and anxiety symptoms $(\mathrm{B}=0.032, p=0.003)$. Poorer subjective sleep quality $(B=0.411, p=0.024)$ and longer sleep latency ( $\mathrm{B}=0.314, p=0.027)$ were both associated with anxiety symptoms (Table 4). Additionally, poorer subjective sleep quality $(\mathrm{B}=0.678, p=0.000)$ and longer sleep latency $(\mathrm{B}=0.447, p=$ 0.002 ) were both associated with depressive symptoms (Table 5).

Our primary hypothesis (Figure 1) was that the association of DED severity, as measured by the OSDI scores, with depressive and anxiety symptoms would be mediated by poorer subjective sleep quality and longer sleep latency (Table 6). In mediation model 1, the effect of DED on anxiety symptoms was positive and significant, with a standardized estimate of 0.141 (95\% CI [0.006$0.048]$ ). This effect was significantly mediated by subjective sleep quality, with a standardized estimate of 0.026 (95\% CI [0.001$0.011])$.

In mediation model 2, the effect of DED on anxiety symptoms was positive and significant, with a standardized estimate of 0.152 (95\% CI [0.006-0.050]). This effect was significantly mediated by sleep latency, with a standardized estimate of 0.015 (95\% CI [0.001-0.008]).

In mediation model 3, the effect of DED on depressive symptoms was positive and significant, with a standardized estimate of 0.126 (95\% CI [0.005-0.046]). This effect was significantly mediated by subjective sleep quality, with a standardized estimate of 0.041 (95\% CI [0.003-0.016]).

In mediation model 4 , the effect of DED on depressive symptoms was positive and significant, with a standardized estimate of 0.147 (95\% CI [0.008-0.050]). This effect was significantly mediated by sleep latency, with a standardized estimate of 0.021 (95\% CI [0.001-0.010]).

In the four models, the confidence interval excluded zero, indicating a significant indirect effect of DED on anxiety and depressive symptoms via mediators.

\section{DISCUSSION}

This study examined the relationship between DED and anxiety and depression, and whether this relationship is mediated by sleep. The results showed that the relationship of DED with anxiety and depression was mediated by subjective sleep quality and sleep latency. Wu et al. (24) found that DED patients with sleep disorders were more likely to be anxious and depressed, which is consistent with our findings.

Eye discomfort symptoms caused by DED may negatively affect mood and mental health $(15,30,31)$.The causal relationship between DED and depression remains unclear. However, some factors have been used to explain this association. First, the two diseases are homologous (32); in particular, both share common risk factors, including female sex and menopause. This suggests that sex hormones play an important role in the development of both diseases. Second, somatization is present in $80 \%$ of patients with depression (33), which may play a role in exacerbating DED symptoms. In addition, we propose two potential mechanisms: subjective sleep quality and sleep latency, which may help explain the relationship of DED with anxiety and depression.

Several studies $(24,34-37)$ have found that DED patients suffer from sleep disorders, short sleep duration, poor sleep quality, insomnia, and sleep apnea. This may be due to the fact that tears are produced by the lacrimal glands, which are innervated by the parasympathetic and sympathetic nerves (38). Sleep disorders have been reported to increase the level of stress hormones, including cortisol, epinephrine, and norepinephrine (39), and decrease parasympathetic activity and increase sympathetic tone. Sleep disorders cause mild activation of the hypothalamic-pituitary-adrenal axis in humans, leading to diuresis and excessive natriuresis $(40,41)$; in addition, 
TABLE 1 | Comparison of demographics in patients with DED With good sleep or poor sleep.

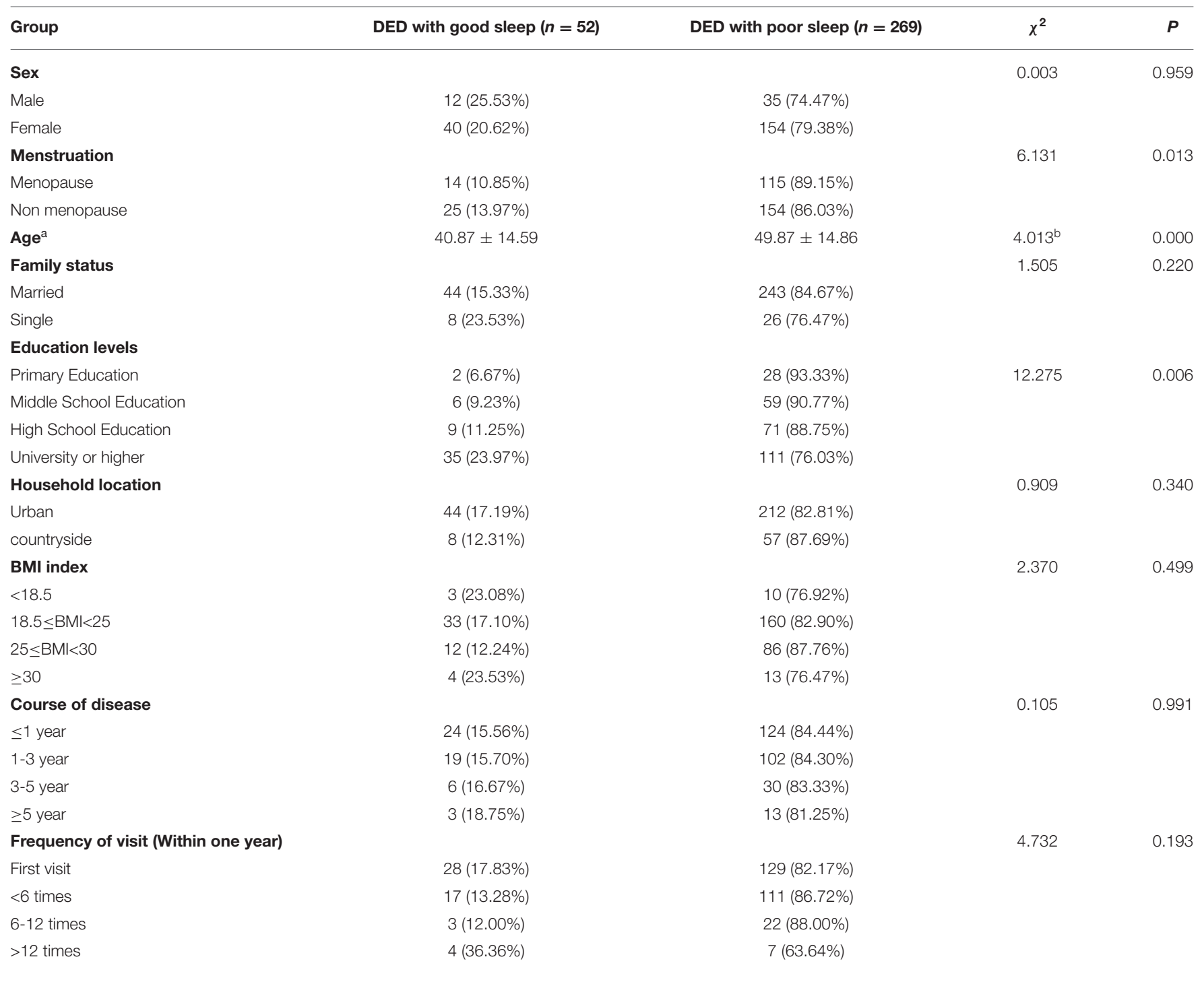

${ }^{a}$ Mean $\pm S D,{ }^{b}$ independent $t$-test.

TABLE 2 | Comparison of anxiety and depression in patients with DED with good or poor sleep.

\begin{tabular}{lccc}
\hline & DED with good sleep $(\boldsymbol{n}=\mathbf{5 2})$ & DED with poor sleep $(\boldsymbol{n}=\mathbf{2 6 9})$ & $\boldsymbol{t}$ \\
\hline Anxiety & $4.75 \pm 3.13$ & $5.90 \pm 3.04$ & -2.475 \\
Depression & $3.96 \pm 2.94$ & $5.75 \pm 3.20$ & -3.738 \\
\hline
\end{tabular}

the circadian rhythm of hormones in the renin-angiotensinaldosterone system is significantly altered (41). It has been suggested that a potential mechanism for dehydration in sleep disorder may involve a reduction in nocturnal blood pressure and a decrease in the renin-angiotensin-aldosterone system hormones (42). Altered levels of these hormones and excess diuresis can thus cause a state of relative dehydration, which can affect tear production.
Subjective sleep quality and latency are important components of sleep. Decreased sleep quality disrupts higherlevel cognitive functions, such as cognitive control $(43,44)$. Cognitive control allows for effective emotion regulation (45), and the ability to regulate emotions provides a critical connection between sleep quality and mood disorders (46). Additionally, when participants with sleep problems view negative emotional pictures, the functional connections between brain regions 
TABLE 3 | Descriptive statistics and intercorrelations between analyzed variables.

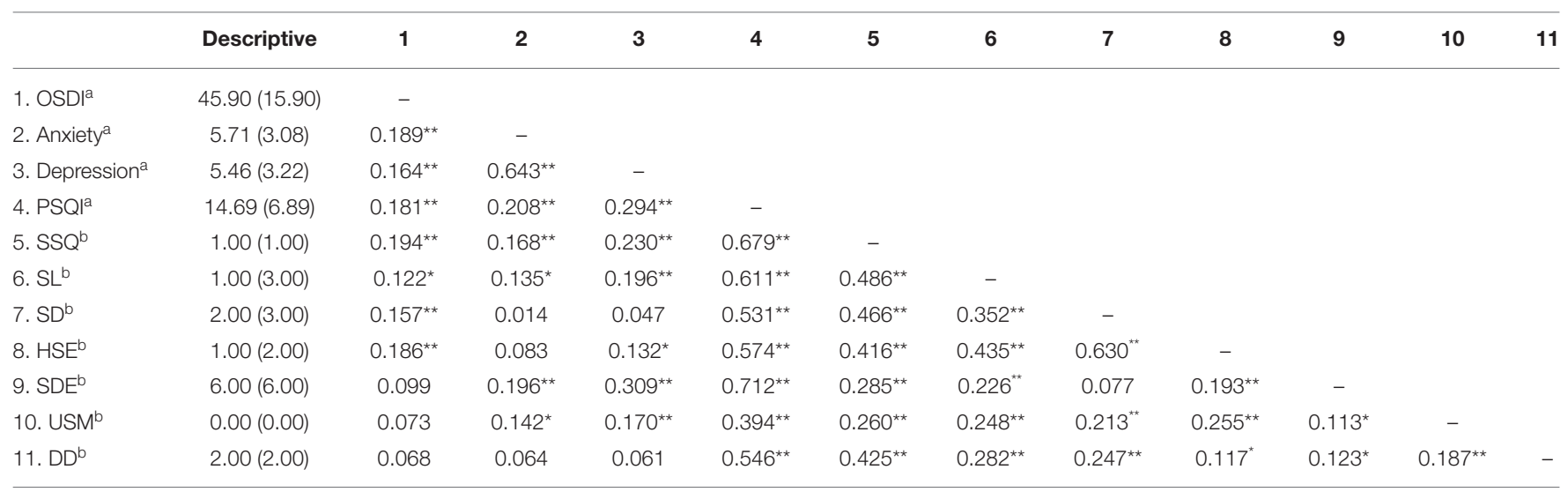

${ }^{a}$ Mean (SD), ${ }^{b}$ Median (IQR), ${ }^{*} P<0.05,{ }^{, *} P<0.01$. SSQ, Subjective Sleep Quality; SL, Sleep Latency; SD, Sleep Duration; HSE, Habitual Sleep Efficiency; SDE, Sleep Disturbance; USM, Used Sleep Medication; DD, Daytime Dysfunction.

TABLE 4 | Mediation analysis of PSQI,SSQ,SL on the relationship between the OSDI and anxiety.

\begin{tabular}{|c|c|c|c|c|c|}
\hline & $B$ & Beta & SE & $t$ & $P$ \\
\hline OSDI on anxiety & 0.032 & 0.167 & 0.011 & 3.030 & 0.003 \\
\hline OSDI on PSQI & 0.085 & 0.197 & 0.024 & 3.581 & 0.000 \\
\hline PSQI on anxiety (indirect effect) & 0.084 & 0.188 & 0.025 & 3.398 & 0.001 \\
\hline OSDI on anxiety (direct effect) & 0.025 & 0.130 & 0.011 & 2.352 & 0.019 \\
\hline OSDI on anxiety & 0.032 & 0.167 & 0.011 & 3.030 & 0.003 \\
\hline OSDI on anxiety (direct effect) & 0.027 & 0.141 & 0.011 & 2.517 & 0.012 \\
\hline OSDI on anxiety & 0.032 & 0.167 & 0.011 & 3.030 & 0.003 \\
\hline OSDI on SL & 0.009 & 0.123 & 0.004 & 2.222 & 0.027 \\
\hline SL on anxiety (indirect effect) & 0.314 & 0.123 & 0.141 & 2.229 & 0.027 \\
\hline OSDI on anxiety (direct effect) & 0.029 & 0.152 & 0.011 & 2.750 & 0.006 \\
\hline
\end{tabular}

SSQ, Subjective Sleep Quality; SL, Sleep Latency.

responsible for cognitive control (medial prefrontal areas) and emotional responses (amygdala) are reduced, resulting in poor individual decision-making over time, such as not seeking support, self-harm, persistent fear and distress, and anxiety and depression (47). Cognitive behavioral therapy for insomnia (CBT-I) includes relaxation, stimulus control, and cognitive therapy (48). Moreover, Ashworth et al. (49) found that CBT-I improved subjective and objective sleep quality and reduced depressive symptoms.

Prolonged sleep latency is considered one of the hallmarks of depression (50). Chronic pain and discomfort in DED may cause central sensitization, a common feature of patients with chronic pain; central sensitization is related to the plasticity of the central nervous system (51), a process in which the nervous system's response is progressively enhanced (52), eventually leading to pain despite low levels of peripheral stimulation. This pain response further prolongs sleep latency and ultimately triggers negative mood, anxiety, depressive symptoms in patients, as has been demonstrated in several studies including breast cancer patients (53). During the COVID-19, people were isolated at home and their use of visual display terminal (VDT) devices increased (54).Increased use of VDT can cause many physical discomforts, such as eye strain, musculoskeletal symptoms, headaches, and sleep problem (55). Individuals who use VDT for more than $6 \mathrm{~h}$ /day are more likely to have sleep problems (56). Some studies have also found a significant correlation between VDT use and difficulty in falling asleep. Reducing the use of VDT may alleviate the symptoms of DED and increase sleep quality.

This study has several limitations. First, we adopted a cross-sectional study, therefore, it was difficult to establish causal relationships between DED, sleep quality, anxiety, and depression. Second, the current data were collected using selfreport scales, with their inherent limitations. Future studies could utilize objective methods, such as polysomnography, to draw more accurate conclusions about sleep outcomes. Third, Galor 
TABLE 5 | Mediation analysis of PSQI,SSQ,SL on the relationship between the OSDI and depression.

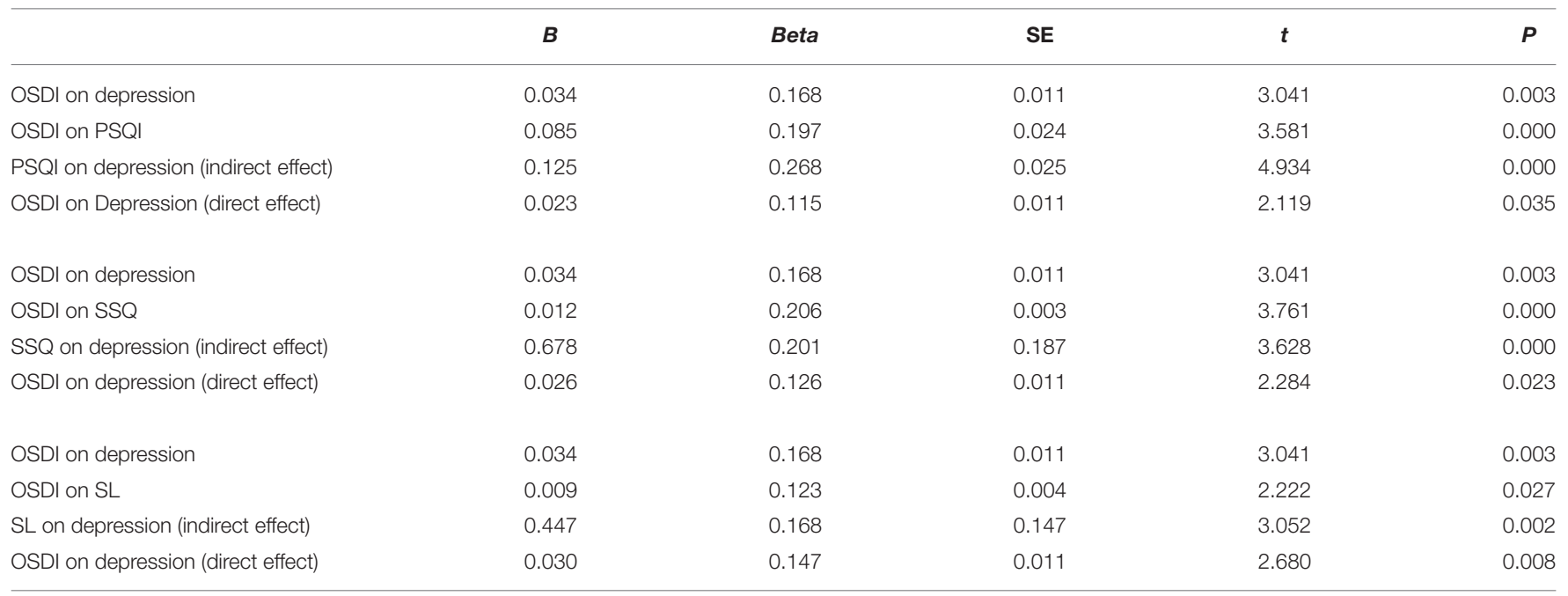

SSQ, Subjective Sleep Quality; SL, Sleep Latency.

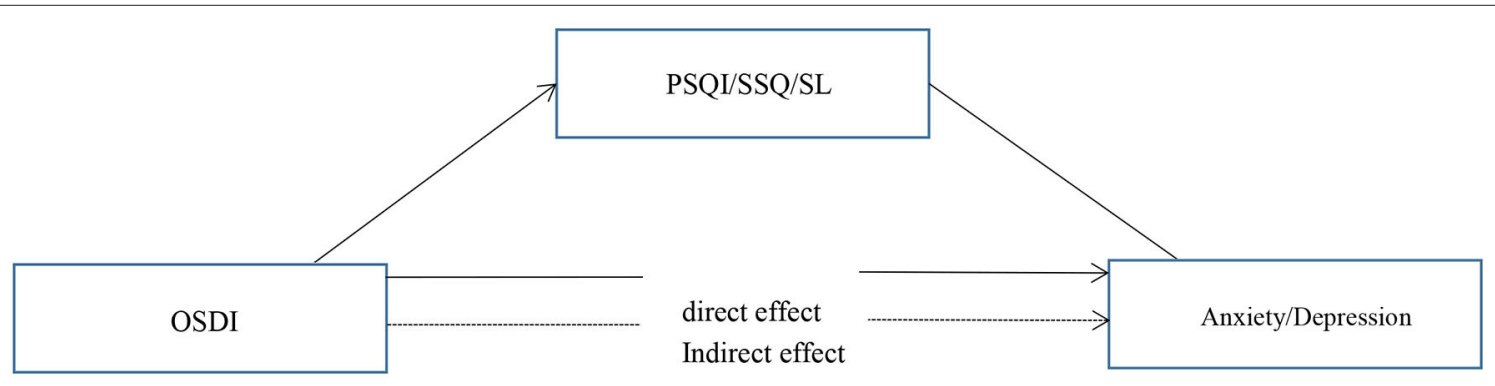

FIGURE 1 | The proposed model of relationships between variables.

TABLE 6 | Bootstrap results for the mediation analysis.

\begin{tabular}{lccc}
\hline Variables & Estimate & SE & LL95\%CL \\
\hline OSDI on anxiety (direct effect) & 0.025 & 0.011 & 0.003 \\
PSQI on anxiety (indirect effect) & 0.007 & 0.003 & 0.046 \\
OSDI on depression (direct effect) & 0.023 & 0.002 & 0.002 \\
PSQI on depression (indirect effect) & 0.011 & 0.115 & 0.014 \\
& & 0.053 & 0.006 \\
OSDI on anxiety (direct effect) & 0.027 & 0.141 & 0.001 \\
SSQ on anxiety (indirect effect) & 0.005 & 0.026 & 0.005 \\
OSDI on depression (direct effect) & 0.026 & 0.003 \\
SSQ on depression (indirect effect) & 0.008 & 0.126 & 0.048 \\
& & 0.041 & 0.011 \\
OSDI on anxiety (direct effect) & 0.029 & 0.046 \\
SL on anxiety (indirect effect) & 0.003 & 0.152 & 0.016 \\
OSDI on depression (direct effect) & 0.030 & 0.015 & 0.001 \\
SL on depression (indirect effect) & 0.004 & 0.147 & 0.008 \\
\hline
\end{tabular}

SSQ, Subjective Sleep Quality; SL, Sleep Latency. 
et al. (36) found that insomnia was more severe in the high pain group of patients with DED. We used the OSDI questionnaire in our assessment of DED, but we did not scale eye pain separately. In future studies we will also focus on eye pain. In addition, the participants in this study were Chinese and constituted an urban community sample from similar areas. Future studies should include larger and more diverse samples.

\section{CONCLUSIONS}

This study showed that many patients with DED experience anxiety, depression, and sleep disorders. Our preliminary research supports subjective sleep quality and sleep latency as mediators between DED and both anxiety and depression. These preliminary findings highlight the need to further explore the role of sleep in the relationship between DED and mood.

\section{DATA AVAILABILITY STATEMENT}

The raw data supporting the conclusions of this article will be made available by the authors, without undue reservation.

\section{REFERENCES}

1. Lin L, Lu L, Cao W, Li T. Hypothesis for potential pathogenesis of SARS-CoV2 infection-a review of immune changes in patients with viral pneumonia. Emerg Microbes Infect. (2020) 9:727-32. doi: 10.1080/22221751.2020.1746199

2. Moghanibashi-Mansourieh A. Assessing the anxiety level of Iranian general population during COVID-19 outbreak. Asian J Psychiatr. (2020) 51:102076. doi: 10.1016/j.ajp.2020.102076

3. Wei N, Huang BC, Lu SJ, Hu JB, Zhou XY, Hu CC, et al. Efficacy of internet-based integrated intervention on depression and anxiety symptoms in patients with COVID-19. J Zhejiang Univ Sci B. (2020) 21:4004. doi: 10.1631/jzus.B2010013

4. Huang Y, Zhao N. Generalized anxiety disorder, depressive symptoms and sleep quality during COVID-19 outbreak in China: a web-based cross-sectional survey. Psychiatry Res. (2020) 288:112954. doi: 10.1016/j.psychres.2020.112954

5. Majumdar P, Biswas A, Sahu S. COVID-19 pandemic and lockdown: cause of sleep disruption, depression, somatic pain, and increased screen exposure of office workers and students of India. Chronobiol Int. (2020) 37:1191200. doi: 10.1080/07420528.2020.1786107

6. Moon JH, Kim KW, Moon NJ. Smartphone use is a risk factor for pediatric dry eye disease according to region and age: a case control study. BMC Ophthalmol. (2016) 16:188. doi: 10.1186/s12886-016-0364-4

7. Sánchez-Valerio MDR, Mohamed-Noriega K, Zamora-Ginez I, Baez Duarte BG, Vallejo-Ruiz V. Dry eye disease association with computer exposure time among subjects with computer vision syndrome. Clinic Ophthalmol. (2020) 14:4311-7. doi: 10.2147/OPTH.S252889

8. Wu P, Duan F, Luo C, Liu Q, Qu X, Liang L, et al. Characteristics of ocular findings of patients with coronavirus disease 2019 (COVID19) in Hubei Province, China. JAMA Ophthalmol. (2020) 138:5758. doi: 10.1001/jamaophthalmol.2020.1291

9. Guan WJ Ni ZY, Hu Y, Liang WH, Ou CQ, He JX, et al. Clinical Characteristics of coronavirus disease 2019 in China. N Engl J Med. (2020) 382:170820. doi: 10.1056/NEJMoa2002032

10. Chen L, Deng C, Chen X, Zhang X, Chen B, Yu H, et al. Ocular manifestations and clinical characteristics of 535 cases of COVID-19 in Wuhan, China: a cross-sectional study. Acta ophthalmologica. (2020) 98:e9519. doi: 10.1111/aos.14472

\section{ETHICS STATEMENT}

The studies involving human participants were reviewed and approved by Medical Ethics Committee of Tianjin Medical University Eye Hospital. The patients/participants provided their written informed consent to participate in this study.

\section{AUTHOR CONTRIBUTIONS}

$\mathrm{QH}, \mathrm{ZC}$, and CX: material preparation, data collection, and analysis were performed. The first draft of the manuscript was written by $\mathrm{QH}$ and all authors commented on previous versions of the manuscript. All authors contributed to the study conception, design, read, and approved the final manuscript.

\section{FUNDING}

This work was supported by a grant from the National Natural Science Foundation of China (Grant No: 81770901).

11. Asiedu K, Dzasimatu SK, Kyei S. Impact of dry eye on psychosomatic symptoms and quality of life in a healthy youthful clinical sample. Eye Contact Lens. (2018) 44:S404-s409. doi: 10.1097/ICL.0000000000000550

12. Sayegh RR Yu Y, Farrar JT, Kuklinski EJ, Shtein RM, Asbell PA, et al. Ocular discomfort and quality of life among patients in the dry eye assessment and management study. Cornea. (2021) 40:86976. doi: 10.1097/ICO.0000000000002580

13. Inomata $T$, Iwagami $M$, Nakamura $M$, Shiang $T$, Fujimoto $K$, Okumura $\mathrm{Y}$, et al. Association between dry eye and depressive symptoms: large-scale crowdsourced research using the DryEyeRhythm iPhone application. Ocul Surf. (2020) 18:312-9. doi: 10.1016/j.jtos.2020.02.007

14. Al-Dairi W, Al Sowayigh OM, Alkulaib NS, Alsaad A. The relationship of dry eye disease with depression in saudi arabia: a cross-sectional study. Cureus. (2020) 12:e12160. doi: 10.7759/cureus.12160

15. Kitazawa M, Sakamoto C, Yoshimura M, Kawashima M, Inoue S, Mimura $\mathrm{M}$, et al. The relationship of dry eye disease with depression and anxiety: a naturalistic observational study. Transl Vis Sci Technol. (2018) 7:35. doi: $10.1167 /$ tvst.7.6.35

16. Yilmaz U, Gokler ME, Unsal A. Dry eye disease and depression-anxietystress: a hospital-based case control study in Turkey. Pak J Med Sci. (2015) 31:626-31. doi: 10.12669/pjms.313.7091

17. van der Vaart R, Weaver MA, Lefebvre C, Davis RM. The association between dry eye disease and depression and anxiety in a large population-based study. Am J Ophthalmol. (2015) 159:470-4. doi: 10.1016/j.ajo.2014.11.028

18. Ayaki M, Kawashima M, Negishi K, Tsubota K. High prevalence of sleep and mood disorders in dry eye patients: survey of 1,000 eye clinic visitors. Neuropsychiatr Dis Treat. (2015) 11:889-94. doi: 10.2147/NDT.S81515

19. Zheng $\mathrm{Y}, \mathrm{Wu} \mathrm{X}$, Lin $\mathrm{X}$, Lin $\mathrm{H}$. The prevalence of depression and depressive symptoms among eye disease patients: a systematic review and meta-analysis. Sci Rep. (2017) 7:46453. doi: 10.1038/srep46453

20. Kim KW, Han SB, Han ER, Woo SJ, Lee JJ, Yoon JC, et al. Association between depression and dry eye disease in an elderly population. Invest Ophthalmol Vis Sci. (2011) 52:7954-8. doi: 10.1167/iovs.11-8050

21. Kaiser T, Janssen B, Schrader S, Geerling G. Depressive symptoms, resilience, and personality traits in dry eye disease. Graefe's archive for clinical and experimental ophthalmology $=$ Albrecht von Graefes Archiv fur klinische und experimentelle Ophthalmologie. (2019) 257:5919. doi: 10.1007/s00417-019-04241-1 
22. Hallak JA, Tibrewal S, Jain S. Depressive symptoms in patients with dry eye disease: a case-control study using the beck depression inventory. Cornea. (2015) 34:1545-50. doi: 10.1097/ICO.0000000000000641

23. Tiskaoglu NS, Yazici A, Karlidere T, Sari E, Oguz EY, Musaoglu M, et al. Dry eye disease in patients with newly diagnosed depressive disorder. Curr Eye Res. (2017) 42:672-6. doi: 10.1080/02713683.2016.1236966

24. Wu M, Liu X, Han J, Shao T, Wang Y. Association between sleep quality, mood status, and ocular surface characteristics in patients with dry eye disease. Cornea. (2019) 38:311-7. doi: 10.1097/ICO.0000000000001854

25. Ayaki M, Kawashima M, Negishi K, Kishimoto T, Mimura M, Tsubota K. Sleep and mood disorders in women with dry eye disease. Sci Rep. (2016) 6:35276. doi: 10.1038/srep35276

26. Roberts RE, Duong HT. The prospective association between sleep deprivation and depression among adolescents. Sleep. (2014) 37:23944. doi: $10.5665 /$ sleep. 3388

27. Salari N, Hosseinian-Far A, Jalali R, Vaisi-Raygani A, Rasoulpoor S, Mohammadi M, et al. Prevalence of stress, anxiety, depression among the general population during the COVID-19 pandemic: a systematic review and meta-analysis. Global Health. (2020) 16:57. doi: 10.1186/s12992-020-00589-w

28. Zigmond AS, Snaith RP. The hospital anxiety and depression scale. Acta Psychiatr Scand. (1983) 67:361-70. doi: 10.1111/j.1600-0447.1983.tb09716.x

29. Yang Y, Ding R, Hu D, Zhang F, Sheng L. Reliability and validity of a Chinese version of the HADS for screening depression and anxiety in psycho-cardiological outpatients. Compr Psychiatry. (2014) 55:21520. doi: 10.1016/j.comppsych.2013.08.012

30. Fine PG. Long-term consequences of chronic pain: mounting evidence for pain as a neurological disease and parallels with other chronic disease states. Pain Med. (2011) 12:996-1004. doi: 10.1111/j.1526-4637.2011.01187.x

31. Na KS, Han K, Park YG, Na C, Joo CK. Depression, stress, quality of life, and dry eye disease in korean women: a population-based study. Cornea. (2015) 34:733-8. doi: 10.1097/ICO.0000000000000464

32. Versura P, Campos EC. Menopause and dry eye. a possible relationship gynecological endocrinology. Offic J Int Soc Gynecol Endocrinol. (2005) 20:289-98. doi: 10.1080/09513590400027257

33. Su KP. Biological mechanism of antidepressant effect of omega-3 fatty acids: how does fish oil act as a 'mind-body interface'? Neurosignals. (2009) 17:14452. doi: 10.1159/000198167

34. Lee W, Lim SS, Won JU, Roh J, Lee JH, Seok H, et al. The association between sleep duration and dry eye syndrome among Korean adults. Sleep Med. (2015) 16:1327-31. doi: 10.1016/j.sleep.2015.06.021

35. Kawashima M, Uchino M, Yokoi N, Uchino Y, Dogru M, Komuro A, et al. The association of sleep quality with dry eye disease: the Osaka study. Clinic Ophthalmol. (2016) 10:1015-21. doi: 10.2147/OPTH.S99620

36. Galor A, Seiden BE, Park JJ, Feuer WJ, McClellan AL, Felix $\mathrm{ER}$, et al. The association of dry eye symptom severity and comorbid insomnia in US Veterans. Eye Contact Lens. (2018) 44:S118-s124. doi: 10.1097/ICL.0000000000000349

37. Matossian C, Song X, Chopra I, Sainski-Nguyen A, Ogundele A. The prevalence and incidence of dry eye disease among patients using continuous positive airway pressure or other nasal mask therapy devices to treat sleep apnea. Clinic Ophthalmol. (2020) 14:3371-9. doi: 10.2147/OPTH.S274949

38. Dartt DA. Neural regulation of lacrimal gland secretory processes: relevance in dry eye diseases. Prog Retin Eye Res. (2009) 28:15577. doi: 10.1016/j.preteyeres.2009.04.003

39. Spiegel K, Leproult R, Van Cauter E. Impact of sleep debt on metabolic and endocrine function. Lancet. (1999) 354:1435-9. doi: 10.1016/S0140-6736 (99)01376-8

40. von Treuer K, Norman TR, Armstrong SM. Overnight human plasma melatonin, cortisol, prolactin, TSH, under conditions of normal sleep, sleep deprivation, and sleep recovery. J Pineal Res. (1996) 20:7-14. doi: 10.1111/j.1600-079X.1996.tb00232.x

41. Mahler B, Kamperis K, Schroeder M, Frøkiær J, Djurhuus JC, Rittig S. Sleep deprivation induces excess diuresis and natriuresis in healthy children. Am J Physiol Renal Physiol. (2012) 302:F236-243. doi: 10.1152/ajprenal.00283.2011
42. McEwen BS. Sleep deprivation as a neurobiologic and physiologic stressor: Allostasis and allostatic load. Metabol Clinic Experiment. (2006) 55:S2023. doi: 10.1016/j.metabol.2006.07.008

43. Goel N, Rao H, Durmer JS, Dinges DF. Neurocognitive consequences of sleep deprivation. Semin Neurol. (2009) 29:320-39. doi: 10.1055/s-0029-1237117

44. Tucker AM, Whitney P, Belenky G, Hinson JM, Van Dongen HP. Effects of sleep deprivation on dissociated components of executive functioning. Sleep. (2010) 33:47-57. doi: 10.1093/sleep/33.1.47

45. Ochsner KN, Gross JJ. The cognitive control of emotion. Trends Cogn Sci. (2005) 9:242-9. doi: 10.1016/j.tics.2005.03.010

46. Mauss IB, Troy AS, LeBourgeois MK. Poorer sleep quality is associated with lower emotion-regulation ability in a laboratory paradigm. Cogn Emot. (2013) 27:567-76. doi: 10.1080/02699931.2012.727783

47. Yoo SS, Gujar N, Hu P, Jolesz FA, Walker MP. The human emotional brain without sleep-a prefrontal amygdala disconnect. Current biology: CB. (2007) 17:R877-878. doi: 10.1016/j.cub.2007.08.007

48. Asarnow LD, Manber R. Cognitive Behavioral Therapy for Insomnia in Depression. Sleep Med Clin. (2019) 14:177-84. doi: 10.1016/j.jsmc.2019.01.009

49. Ashworth DK, Sletten TL, Junge M, Simpson K, Clarke D, Cunnington D, et al. A randomized controlled trial of cognitive behavioral therapy for insomnia: an effective treatment for comorbid insomnia and depression. J Couns Psychol. (2015) 62:115-23. doi: 10.1037/cou0000059

50. Rotenberg VS, Shami E, Barak Y, Indursky P, Kayumov L, Mark M, et al. sleep latency and wakefulness in the first sleep cycle as markers of major depression: a controlled study vs. schizophrenia and normal controls Progress in neuro-psychopharmacology \& biological. Psychiatry. (2002) 26:1211-5. doi: 10.1016/S0278-5846 (02)00216-6

51. Latremoliere A, Woolf CJ. Central sensitization: a generator of pain hypersensitivity by central neural plasticity. J Pain. (2009) 10:895926. doi: 10.1016/j.jpain.2009.06.012

52. Mantyh PW, Clohisy DR, Koltzenburg M, Hunt SP. Molecular mechanisms of cancer pain. Nat Rev Cancer. (2002) 2:201-9. doi: 10.1038/nrc747

53. Kirsch JL, Robinson ME, McCrae CS, Kacel EL, Wong SS, Patidar S, et al. Associations among sleep latency, subjective pain, and thermal pain sensitivity in gynecologic cancer. Pain Med. (2020) 21:5-12. doi: 10.1093/pm/pny236

54. Cartes C, Segovia C, Salinas-Toro D, Goya C, Alonso MJ, Lopez-Solis R, et al. Dry eye and visual display terminal-related symptoms among university students during the coronavirus disease pandemic. Ophthalmic Epidemiol. (2021) 21:1-7. doi: 10.1080/09286586.2021.1943457

55. Nakazawa T, Okubo Y, Suwazono Y, Kobayashi E, Komine S, Kato N, et al. Association between duration of daily VDT use and subjective symptoms. Am J Ind Med. (2002) 42:421-6. doi: 10.1002/ajim.10133

56. Giahi O, Shahmoradi B, Barkhordari A, Khoubi J. Visual display terminal use in iranian bank tellers: effects on job stress and insomnia. Work. (2015) 52:657-62. doi: 10.3233/WOR-152190

Conflict of Interest: The authors declare that the research was conducted in the absence of any commercial or financial relationships that could be construed as a potential conflict of interest.

Publisher's Note: All claims expressed in this article are solely those of the authors and do not necessarily represent those of their affiliated organizations, or those of the publisher, the editors and the reviewers. Any product that may be evaluated in this article, or claim that may be made by its manufacturer, is not guaranteed or endorsed by the publisher.

Copyright (c) $2022 \mathrm{He}$, Chen, Xie, Liu and Wei. This is an open-access article distributed under the terms of the Creative Commons Attribution License (CC BY). The use, distribution or reproduction in other forums is permitted, provided the original author(s) and the copyright owner(s) are credited and that the original publication in this journal is cited, in accordance with accepted academic practice. No use, distribution or reproduction is permitted which does not comply with these terms. 